\title{
Immunogenicity and biomarkers: bioanalytical challenges and considerations
}

\author{
George R Gunn ${ }^{*}, 1$, Christopher Evans ${ }^{1}$ \& Eric Yang ${ }^{1}$ \\ ${ }^{1}$ Bioanalysis, Immunogenicity \& Biomarkers, GlaxoSmithKline Pharmaceutical R\&D 709 Swedeland Rd, King of Prussia, PA 19406, \\ USA \\ * Author for correspondence: george.x.gunn@gsk.com
}

"Assay multiplicity and complexity generally requires analytical staff to possess a certain amount of scientific nimbleness, in other words, the ability to apply a variety of formats or assay platforms to address the required analytical end points."

First draft submitted: 24 August 2017; Accepted for publication: 15 September 2017; Published online: 9 November 2017

Keywords: bioanalytical • biomarkers $\bullet$ immunogenicity $\bullet$ interference $\bullet$ resourcing $\bullet$ sensitivity

In drug development, particularly for biotherapeutics, analytical assessments of immunogenicity and biomarkers are two sides of the same coin. Both work closely with pharmacokinetic (PK) assessments to provide analytical evidence or correlations with drug efficacy and safety measures. While immunogenicity assessments are commonly qualitative or quasi-quantitative and biomarker analytic readouts are usually quantitative, both typically utilize similar analytical platforms. A significant focus on method quality and experimental validation is warranted to meet scientific and regulatory expectations due to the clinical importance and labeling implications of immunogenicity and biomarker assessments related to therapeutic efficacy and safety.

Preclinical and clinical assessments of biotherapeutic immunogenicity (i.e., detection and characterization of immune responses against a biotherapeutic or related protein) are critical to help define safety profiles of these products. Additionally, immune responses may impact pharmacokinetics, potentially either accelerating or impeding clearance and as a consequence, efficacy may be altered. Through various industry working groups, white papers and agency guidance's the bioanalytical characterization of these events are well described [1-3].

Equally important in the development of biotherapeutics and new chemical entities, is the characterization of pharmacology-relevant biomarkers to demonstrate proof of mechanism and help guide clinical dose selection. The 'three pillars of survival' concept has evolved to apply bioanalytical tools used to explore exposure at the site of action, target binding and expression of functional pharmacological activity [4]. Similarly, the experimental design of these investigations is well described [5-7].

The training and expertise of analytical staff, as well as the instrumentation and technical platforms used for immunogenicity and biomarker characterizations are nearly identical. Therefore, substantial overlap exists in bioanalytical challenges and considerations for both determinations. This article explores several major themes common to both types of determinations, including: sensitivity, interference (e.g., drug, target and anti-drug antibodies $[\mathrm{ADA}])$, method complexity and resourcing.

\section{Sensitivity}

Potentially the most critical component of any bioanalytical assay is sensitivity, demonstrating that the method is capable of detecting an analyte at concentrations relevant to therapeutics pharmacology, mode of action and safety. While mass spectrometry is growing in its application for biomarker and immunogenicity assessment, the predominant platforms for these determinations are generally immunoassays. Various formats can be used, whether direct, indirect, bridging or competitive; however, the assays must be designed to specifically detect clinically relevant ADA or circulating biomarker concentrations. Immunoassay platforms (e.g., ELISA and electrochemiluminescence immunoassay) typically provide sufficient sensitivity for immunogenicity assessments, rarely requiring sub-ng/ml sensitivity to identify clinically relevant ADA. Additionally, gains in ADA assay sensitivity 
often translate directly into reduced drug tolerance. Hence, fit-for-purpose ADA assay development often requires some balancing to achieve sufficient sensitivity and drug tolerance. However, very high sensitivity methods are becoming more commonly required to detect and measure subsets of biomarkers. These very high sensitivity biomarker assays ( $\leq \mathrm{pg} / \mathrm{ml}$ range) may require use of platforms (i.e., Singluex Erenna ${ }^{\circledR}$ or Quanterix Simoa) less robust than more standard immunoassays, where users can experience high run and sample failure rates, many times upwards of $30 \%$. Use of these more specialized platforms may also restrict option if externalized sample testing is desired. Therefore, potential gains in sensitivity may come at a cost of operational efficiency that may limit testing capacity and opportunities to outsource bioanalytical efforts. It is critical to retain sufficient specificity and selectivity as sensitivity limits are pushed toward the lower end of analytical limits, regardless of the platform used. In general, for immunogenicity and biomarker methods, assay requirements must be defined prior to development, to ensure the desired balance of key assay parameters is maintained.

\section{Interference}

Once an appropriate platform and assay range have been established, additional focus must be placed on limiting drug interference. Residual drug levels in samples tested for ADAs or biomarkers may interfere with the detection of an analyte. In the case of immunogenicity analysis, demonstrating sufficient method of drug tolerance to enable detection of control ADAs in the presence of expected trough levels of drug has been a regulatory expectation for nearly a decade. Common approaches to improve drug tolerance frequently utilize acid dissociation [8] or prolonged incubation (possibly with expanded exploration of minimal required dilution [9]), are often required to develop methods with sufficient resistance to drug interference. Debate continues regarding the most appropriate approach to achieve sufficient drug tolerance and whether the improvement of drug tolerance may have complicated detection of clinically relevant ADAs [10]. Key points of consideration surround the potential risk that acid dissociation may influence detection of weakly binding or acid sensitive ADA and whether increased detection of ADAs (many of which may have titers insufficient to impact drug level or efficacy), may complicate identification of more clinically relevant $\mathrm{ADA}$ responses that may be more obvious from less drug tolerant methods that only detect ADA capable of accelerating drug clearance. In addition, the widespread use of acid dissociation to improve detection of ADAs in the presence of drugs has contributed to the challenge of target interference, whereby accumulated drug target (more frequently observed with multimeric soluble targets) released from the drug by the acid treatment interferes in the accurate detection of ADAs by the commonly used immunoassay formats applied [11].

Drug levels and ADAs in the matrix may potentially interfere in biomarker assays, particularly in assays measuring target engagement and receptor occupancy. Assessments of interference should be explored during method development and a risk-based approach should be used to mitigate or eliminate interference to a sufficient degree that appropriate levels of confidence can be attributed to the analytical results. These interferences have become one of the major challenges in developing suitable assays; therefore, alternative methodologies such as precipitation and acid dissociation (PandA) have evolved and been shown to be successful in certain situations [12].

In addition to target interferences, the development of antibodies and reagents suitable for immunoassay methods can be very costly and time consuming; hence, the rise of ligand or reagent free approaches such as LC-MS/MS using tryptic digestion. The use of immunoaffinity enrichment followed by mass spectrometric analysis has also seen a surge in popularity. Recent sensitivity advances of this platform have enabled application for both ADA and biomarker characterization (often in the $\mathrm{pg} / \mathrm{ml}$ range). However, the utility of LC-MS/MS is primarily applied early in the clinical development, mostly due to higher cost associated with longer analysis time and smaller sample throughput compared with immunoassay methods. A future perspective that one can envision is the use of LCMS/MS in early development when sample numbers are limited and high quality reagents are not widely available with subsequent transition into immunoassay platforms later in development to support larger clinical trials.

\section{Method complexity}

Compared to PK analysis (where a single assay is required to sensitively, accurately and precisely measure a single analyte), immunogenicity and biomarker assessments commonly require suites of assays to detect and adequately characterize ADA or PD markers to support assessments of efficacy and/or safety. Immunogenicity analytical tools are applied in a risk-based manner with standard assessments, which typically include: screening, confirmatory, titer and neutralizing antibody assays, but which may also be expanded to include: domain mapping, cross-reactivity testing or isotyping depending on the risk of immune consequences. Similarly, biomarker assessments commonly include measurement of free, total and bound drug. Additionally, various isoforms of biomarkers may require 
exploration. All of these factors multiply the complexity of developing, validating, applying, and outsourcing immunogenicity and biomarker assays. Consideration is warranted if some biomarker and/or immunogenicity assessments may have a level of redundancy. For example, with sufficient scientific justification, sensitive and reliable PD markers may be considered for substitution in the place of neutralizing antibody assays, which are frequently cell-based, notoriously susceptible to interference, often have limited dynamic range and are typically less sensitive than ADA detection methods.

Further confounding our ability to adequately support late stage development are the challenges experienced during assay transfers. Even when identical reagent batches and plates are used, there are often issues which arise during internal intra group transfers as well as from originator to CRO. In general, science is facing a 'reproducibility crisis' [13]. Common questions are: 'how do we even trust the data if the assay cannot be reproduced in another lab?' or 'our methods are 'validated', therefore are they easily replicable?' In order to help alleviate and combat these concerns, researchers have put in place robust cross-validation procedures and rely on the support from trusted and preferred CRO providers in order to ensure that methods can be reliably transferred.

Another area of emerging interest is at-home sampling and measurements. This undoubtedly challenges the current model of central bioanalytical labs, where biological samples are collected and shipped in batches for analysis and the data becomes available after a few days/weeks later following their analysis. Home sampling and measurements (e.g., immunogenicity or biomarker) holds a lot of promise as it provides doctors and/or patients real time data that may enable them to react quickly in the event of severe adverse effects or lack of drug efficacy.

\section{Resourcing}

Assay multiplicity and complexity generally requires analytical staff to possess a certain amount of scientific nimbleness, in other words, the ability to apply a variety of formats or assay platforms to address the required analytical end points. Given the regulatory guidance, industry white papers, and the regulated nature typically applied to immunogenicity and biomarker analyses, areas of overlap exist in the documentation, quality and method validation practices. Hence, there are substantial similarities in the skill and knowledge sets required to effectively develop and implement immunogenicity and biomarker assay methods. While this suggests that resourcing immunogenicity and biomarker assay development, validation or implementation may draw from the same talent pool, assay complexity and multiplicity makes outsourcing these assays more challenging from a scientific management perspective. In addition, externalizing analysis of immunogenicity and biomarker assay sets is frequently more expensive due to time and complexity (i.e., multiple biomarker end points and/or tiered strategy for immunogenicity assessment) compared with analogous PK measurements.

\section{Conclusion}

In conclusion, as immunogenicity and biomarker assessments, assay platforms and regulatory expectations have evolved and matured, new challenges continue to arise. The complexities and similarities of the two disciplines regarding assay multiplicity, achieving sufficient method sensitivity and engineering adequate tolerance to interference contribute to the time and cost involved in generating these end points necessary for assessing drug efficacy and safety. However, the similarities in assay platform, scientific training required for analytical scientists and the benefit of cross-trained and scientifically nimble staff continues to provide opportunities to orchestrate efficiencies in the application of immunogenicity and biomarker research.

\section{Financial \& competing interests disclosure}

The authors have no relevant affiliations or financial involvement with any organization or entity with a financial interest in or financial conflict with the subject matter or materials discussed in the manuscript. This includes employment, consultancies, honoraria, stock ownership or options, expert testimony, grants or patents received or pending, or royalties.

No writing assistance was utilized in the production of this manuscript.

\section{References}

1. Guidance for Industry Immunogenicity Assessment for Therapeutic Protein Products (2014). www.fda.gov/downloads/drugs/guidances/ucm338856.pdf

2. Guideline on Immunogenicity assessment of therapeutic proteins (EMEA/CHMP/BMWP/14327/2006 Rev 1) (2017). www.ema.europa.eu/docs/en_GB/document_library/Scientific_guideline/2017/06/WC500228861.pdf 
3. Shankar G, Arkin S, Cocea L et al. Assessment and reporting of the clinical immunogenicity of therapeutic proteins and peptides-harmonized terminology and tactical recommendations. AAPS J. 16(4), 658-673 (2014).

4. Morgan P, Van Der Graaf PH, Arrowsmith J et al. Can the flow of medicines be improved? Fundamental pharmacokinetic and pharmacological principles toward improving Phase II survival. Drug Discov. Today 17(9-10), 419-424 (2012).

5. Liang M, Schwickart M, Schneider AK et al. Receptor occupancy assessment by flow cytometry as a pharmacodynamic biomarker in biopharmaceutical development. Cytometry B Clin. Cytom. 90(2), 117-127 (2016).

6. Wagner JA. Strategic approach to fit-for-purpose biomarkers in drug development. Annu. Rev. Pharmacol. Toxicol. 48, 631-651 (2008).

7. Xu Z, Davis HM, Zhou H. Clinical impact of concomitant immunomodulators on biologic therapy: pharmacokinetics, immunogenicity, efficacy and safety. J. Clin. Pharmacol. 55(Suppl. 3), S60-S74 (2015).

8. Smith HW, Butterfield A, Sun D. Detection of antibodies against therapeutic proteins in the presence of residual therapeutic protein using a solid-phase extraction with acid dissociation (SPEAD) sample treatment prior to ELISA. Regul. Toxicol. Pharmacol. 49(3), 230-237 (2007).

9. Mire-Sluis AR, Barrett YC, Devanarayan V et al. Recommendations for the design and optimization of immunoassays used in the detection of host antibodies against biotechnology products. J. Immunol. Methods 289(1-2), 1-16 (2004).

10. Lu Y, Khawli LA, Purushothama S, Theil FP, Partridge MA. Recent advances in assessing immunogenicity of therapeutic proteins: impact on biotherapeutic development. J. Immunol. Res. 2016, 8141269 (2016).

11. Dai S, Schantz A, Clements-Egan A, Cannon M, Shankar G. Development of a method that eliminates false-positive results due to nerve growth factor interference in the assessment of fulranumab immunogenicity. AAPS J. 16(3), 464-477 (2014).

12. Zoghbi J, Xu Y, Grabert R, Theobald V, Richards S. A breakthrough novel method to resolve the drug and target interference problem in immunogenicity assays. J. Immunol. Methods 426, 62-69 (2015).

13. Baker M. 1500 scientists lift the lid on reproducibility. Nature 533(7604), 452-454 (2016). 